\title{
Instrumentos económicos y de política pública para la asignación de agua subterránea para uso agrícola en México
}

\author{
Edgar Rivero Cob \\ Investigador del Instituto Nacional de Ecología \\ eriverocob@yahoo.com.mx
}

Helena García Romero Insvestigadora de la Fundación para la Educación Superior y el Desarrollo de Colombia hgarcía@fedesarrollo.org.co 


\section{RESUMEN}

Uno de cada tres de los acuíferos más importantes de México se encuentra sobreexplotado. Esto significa que cada año se extrae más agua de la que la naturaleza recarga vía precipitación e infiltración. El principal usuario de agua subterránea es el sector agrícola, con $77 \%$ del agua concesionada. Sin embargo, su uso no es sustentable, solo $46 \%$ de los usuarios tienen alguna tecnología de riego ahorradora de agua. Hay dos causas que explican este uso no sustentable del agua en las actividades agrícolas: el subsidio a las tarifas eléctricas para el bombeo de agua y los fallos institucionales en la asignación, inspección, monitoreo y sanción de las concesiones por usar el agua. Los dos fallos de política pública afectan directamente los incentivos generados por los mecanismos vigentes de asignación de agua subterránea, a saber, los precios directos con medición volumétrica, los precios indirectos acoplados al consumo de energía y los mercados de transmisiones de derechos de agua. El objetivo de este trabajo es: a) analizar conceptualmente los incentivos que se generan a partir de la forma de asignación de agua subterránea vigente, mediante un mecanismo vía cantidades (concesiones), b) identificar las ganancias en sustentabilidad de los acuíferos, de una reforma institucional que transite hacia un mecanismo de asignación vía precios (tarifas) y c) quiénes serían los ganadores y perdedores de esta reforma potencial.

Palabras clave: agua subterránea, economía política, mecanismos de precios, sobreexplotación de acuíferos

Clasificación JEL: L5, Q15, Q31, Q52, H23 


\begin{abstract}
One third of Mexico's aquifers are overexploited. One of the main drivers of overexploitation is the excessive extraction of groundwater for agricultural purposes. The agricultural sector uses 77 per cent of concessioned water with an average irrigation efficiency of 46 per cent. There are two main causes for excessive extraction: the subsidized electricity fees for agricultural groundwater pumping and institutional weakness in assigning, monitoring and enforcing water concessions. These policy failures do not send the correct scarcity signals and do not provide incentives for a more efficient use of water.

This paper analyzes the political economy associated with the current quantity allocation mechanism and evaluates an institutional reform towards an indirect pricing mechanism as well as the associated sustainability gains for the aquifers due to a decrease in the groundwater extraction rate.
\end{abstract}

Keywords: groundwater, political economy, pricing mechanisms, aquifer's overexploitation.

JEL Clasification: L5, Q15, Q31, Q52, H23 


\section{INTRODUCCIÓN}

En México existe una crisis de sobreexplotación de acuíferos. Anualmente en 101 de los 282 acuíferos más importantes del país se extrae más agua de la que la naturaleza recarga a través de la precipitación e infiltración. El número se ha triplicado entre 1975 y 2007 (en 1975 solamente 32 acuíferos se encontraban sobreexplotados) (Conagua, 2008). Por su estrecha vinculación con el agua superficial, esta situación genera problemas de cambios en el volumen de los caudales, y en su dinámica de abastecimiento a lo largo del año, deterioro de los sistemas semiacuáticos (i.e. humedales), intrusión salina, subsidencia de tierras y deterioro de la calidad de agua, entre otros (Muñoz, 2007). La extracción excesiva de agua subterránea en el presente genera también problemas de abasto y externalidades negativas para las generaciones futuras de agricultores, industriales y usuarios domésticos, quienes se verán forzados a bombear agua de mayores profundidades y posiblemente de menor calidad o a buscar fuentes alternativas con un mayor costo de suministro.

El sector agrícola es el principal consumidor de agua subterránea; el porcentaje del agua concesionada del país que utiliza es mayor al promedio mundial (72\%) y al de otros países con más disponibilidad hídrica y con un tamaño de economía similar, pero con una mayor aportación de esta actividad a su PIB nacional. Por ejemplo, en Brasil el sector agrícola representa $7 \%$ del PIB y utiliza $67 \%$ de agua, mientras que en México el sector representa 4\% del PIB y consume $77 \%$ del agua subterránea concesionada (Conagua, 2008; FAO, 2007). Además, más de las dos terceras partes del agua que se extrae de los acuíferos del país se utilizan con eficiencias promedio de riego de $46 \%$ (Presidencia de la República, 2007), es decir, casi la mitad del agua que se extrae de los pozos para riego agrícola se infiltra o se evapora. Adicionalmente, de acuerdo con los censos de aprovechamientos de la Comisión Nacional del Agua, Conagua, 2010, 48\% del agua de los agricultores es ilegal, es decir, no cuenta con una concesión, y del porcentaje que es legal, sólo $39 \%$ tiene un medidor para controlar y monitorear el consumo de agua. Por su parte, Muñoz (2007) encuentra que ocho de cada diez 
beneficiarios con medidor del programa de uso eficiente del agua y la energía eléctrica de Conagua ${ }^{1}$ extraen un volumen de agua mayor al volumen concesionado.

La literatura disponible para México (Muñoz, 2007; Guevara, 2008) documenta que la sobreexplotación de los acuíferos de México por uso agrícola es causada por dos fallas de política pública: por un lado, no existe un cumplimiento óptimo de la Ley de Aguas Nacionales (LAN) que evite la extracción ilegal sin concesiones, ni aquella que ocurre por encima del volumen concesionado; por otro lado, el gobierno federal subsidia la extracción de agua vía la reducción de tarifas eléctricas de bombeo, lo que provoca un aumento de la cantidad de agua que se saca cada año, al no haber una señal de escasez vía precios. Estos errores de política pública ocasionan que los tres mecanismos vigentes de asignación: 1) precio directo con medición volumétrica, 2) precio indirecto de la energía eléctrica consumida para extraer agua y 3) mercado de transmisiones de derechos de agua, den resultados subóptimos en términos de eficiencia, que se reflejan en los niveles de extracción del agua subterránea y en la sobreexplotación de acuíferos.

En las siguientes secciones describiremos la estrategia de política pública para hacer frente al problema de escasez de aguas nacionales, el uso que se ha hecho de estos tres mecanismos y sus resultados, así como una posible reforma y sus efectos distributivos.

\section{LOS INSTRUMENTOS DE LA POLÍTICA HÍDRICA NACIONAL}

Históricamente la Conagua ha tratado de resolver el problema de escasez de aguas nacionales con el aumento de la oferta, a través del impulso de programas y el incremento del presupuesto para desarrollar suministros

\footnotetext{
${ }^{1}$ El Programa de uso eficiente del agua y la energía eléctrica (PUEAEE) se origina en 1990 y busca propiciar el ahorro del agua y la energía eléctrica mediante la rehabilitación de plantas de bombeo, de pozos y equipos de bombeo para riego agrícola localizados en las unidades de riego, así como de los pozos particulares ubicados dentro de los distritos de riego.
} 
de agua adicionales, en lugar de analizar si los suministros de agua existentes se están utilizando de manera eficiente (Roemer, 2007). Por ejemplo, de acuerdo con la Agenda del agua 2030 (Conagua, 2011), para hacer frente a un escenario futuro de escasez de agua causado por menor precipitación, menor recarga de los mantos acuíferos y por un aumento de la temperatura y la evapotransporación, se requeriría aumentar la eficiencia de riego en 17 puntos porcentuales (pasar de un promedio de 46 a 63\%). Como se muestra en el cuadro 1, para Conagua esto implicaría aumentar la superficie de riego a 5.8 millones de hectáreas ${ }^{2}$ (actualmente son 800 mil) e invertir 51 mil millones de pesos en los próximos 20 años. Ahora bien, esa inversión probablemente sea mayor, ya que el escenario no toma en cuenta un aumento en la demanda de agua para el sector agrícola. Así las cosas, Conagua asocia el concepto de sustentabilidad con mayor gasto público en infraestructura de riego, y no con la administración de las fuentes de agua hoy disponibles.

Sin embargo, como se verá más adelante, para poder hacer frente a las condiciones futuras de escasez es necesario administrar también la demanda, ya que aún con inversiones cuantiosas y bajo el supuesto que la oferta de agua es perfectamente elástica, difícilmente se logrará la meta de sustentabilidad en el uso del agua en el sector agrícola. Asimismo, el uso de políticas e instrumentos de expansión de la oferta de agua, a través del desarrollo de nuevas fuentes de abastecimiento de agua respecto a las ya disponibles (i.e. construcción de presas, acueductos, etc.) va en sentido contrario a la orientación de los esfuerzos internacionales de reforma en una era de escasez: la administración de la demanda de agua, esto es, incentivar el uso eficiente del agua ya disponible.

\footnotetext{
${ }^{2} \mathrm{Si}$ asumimos que toda la nueva superficie de riego incorporada hace uso de tecnologías de riego eficientes.
} 


\section{Cuadro 1}

Prospectiva de la inversión en infraestructura para riego agrícola en México (2002 -2025)

\begin{tabular}{|l|l|l|l|}
\hline \multirow{2}{*}{ Concepto } & \multicolumn{3}{c|}{ Escenario } \\
\cline { 2 - 4 } & Actual & Tendencial & Sustentable \\
\hline Hectáreas de riego modernizadas (millones de has) & 0.8 & 1.1 & 5.8 \\
\hline Eficiencia en el uso del agua (\% de pérdidas) & $54 \%$ & $51 \%$ & $37 \%$ \\
\hline Distritos de riego & $62 \%$ & $57 \%$ & $45 \%$ \\
\hline Unidades de riego & $45 \%$ & $44 \%$ & $26 \%$ \\
\hline Demanda global de agua (Mm3) & 56210 & 58098 & 58346 \\
\hline Inversión anual en el sector (miles de millones de pesos) & 14 & 16 & 30 \\
\hline
\end{tabular}

Fuente: Agenda del agua 2030, Conagua (2011)

Por ejemplo, a pesar de las inversiones en infraestructura hidráulica autorizadas y proyectadas, de acuerdo con la información proveniente del Censo agrícola y ganadero, 2007, del Instituto Nacional de Estadística, Geografía e Informática, INEGI, se calculó que en 15 de los acuíferos más sobreexplotados para el país, ${ }^{3}$ solo $25 \%$ de las unidades de producción rurales tienen riego tecnificado (i.e. goteo), mientras que $41 \%$ utilizan la técnica de riego más desperdiciadora de agua: canales de tierra.

Asimismo, a pesar de que existen al menos cuatro programas federales ${ }^{4}$ de la Secretaría de Agricultura, Ganadería, Desarrollo Rural, Pesca y Alimentación, Sagarpa, y Conagua, con un presupuesto autorizado en 2010 de más de 9 mil millones de pesos para apoyar la tecnificación, 50\% de los agricultores que desean invertir en la modernización de sus parcelas no han podido hacerlo en los últimos seis años (INE-UACH, 2010). Según

\footnotetext{
${ }^{3}$ Maneadero y San Quintín (Baja California), Navidad-Potosí-Raíces (Nuevo León), Valle de Tecamachalco (Puebla), Aguanaval, Calera, Chupaderos, Loreto y Ojo Caliente (Zacatecas), Valle de Aguascalientes, Valle de Chicalote, El Llano (Aguascalientes), Vanegas-Catorce (San Luis Potosí) y Los Chirriones (Sonora).

${ }^{4}$ Programa de rehabilitación y modernización de distritos de riego, Programa de modernización y tecnificación de unidades de riego y Programa de desarrollo parcelario, de Conagua, y el Proyecto estratégico de tecnificación de riego agrícola de Sagarpa.
} 
la encuesta a usuarios agrícolas de tarifas eléctricas 2010, la principal razón por la cual no han podido tecnificarse los agricultores interesados es la falta de recursos propios para cumplir con la contraparte requerida por los programas ofrecidos.

Esto parece mostrar un primer indicio de que la intervención pública, que busca incidir por el lado de la oferta, no es suficiente para llevar a una asignación eficiente de los recursos hídricos. Es importante mencionar, sin embargo, que, en cuanto al ahorro del recurso, la administración de la oferta no es del todo opuesta a la administración de la demanda. El uso de ciertos tipos de ingeniería hidráulica (construcción de presas, perforación de pozos, instalación de tuberías, etc.) para aumentar las fuentes de abastecimiento de agua para uso agrícola puede, eventualmente, propiciar el ahorro de agua, al evitar fugas y desperdicios a través de la disminución de la evapotranspiración del agua destinada hacia los cultivos. Lo que hace la diferencia entre uno y otro tipo de instrumentos es la eficiencia y la efectividad con la que se alcanzan los resultados en un contexto de restricción presupuestal.

\subsection{Mecanismos de precios para la administración de la demanda}

Para lograr una adecuada administración de la demanda de agua existen varios mecanismos. El más comúnmente estudiado es el mecanismo de precios, cuyo papel fundamental es ayudar a asignar los recursos escasos entre usos y usuarios que compiten entre sí. Una manera de alcanzar una asignación eficiente del agua es establecer un precio correcto a su consumo, de modo que se asigne de acuerdo con quienes valoren más el bien o servicio.

Existen varios mecanismos de establecimiento de precios al agua para riego agrícola, los más utilizados son: a) métodos de medición volumétrica; b) métodos de medición no volumétrica y c) métodos basados en el mercado de derechos de agua (Tsur et al., 2004). Los tres mecanismos de asignación difieren en las instituciones e información que requieren para su funcionamiento e instrumentación, así como en la eficiencia y equidad de sus resultados. 
a) Métodos de medición volumétrica: requieren de dispositivos para medir el consumo de agua de cada usuario. Los costos de su instrumentación son relativamente altos, ya que necesitan que la autoridad central del agua o asociaciones de usuarios de agua establezcan el precio, monitoreen y recolecten tarifas o cuotas.

b) Métodos de medición no volumétrica: pueden utilizarse en situaciones en las que el mecanismo de precios de medición volumétrica no es factible o deseable (altos costos de instrumentación que no alcanzan a igualar o que superan los beneficios sociales). Aquí los precios se establecen de manera indirecta a través del producto obtenido, algún insumo de producción, el área irrigada o una cuota fija. Si bien el precio por área es el método más común para el establecimiento de precios para el agua de riego agrícola con medición no volumétrica, el precio de la energía (electricidad o diesel) para extraer agua del subsuelo puede ser una herramienta poderosa para influir en el consumo de agua, ya que es el principal costo variable en la extracción de la misma (Scott, Shah y Buechler, 2003; Kemper et al., 2003). Paradójicamente, en muchas áreas del mundo los precios de la energía no pueden ser utilizados como mecanismo para enviar señales de escasez del recurso, ya que se destinan enormes subsidios para disminuir los costos privados de extracción de los agricultores.

c) Métodos basados en el intercambio de derechos de agua: un mercado de agua es un arreglo en el cual los que poseen los derechos de acceso al agua subterránea los comercian o transmiten entre ellos o con partes externas. El mercado del agua no es otra cosa que un conjunto de arreglos que permiten que el agua sea intercambiada de manera voluntaria, parcial o totalmente, y temporal o definitivamente. Los intercambios de derechos de agua han sido ampliamente reconocidos como mecanismos de asignación que pueden llevar a ganancias en eficiencia y conservación, ya que proveen un mecanismo más flexible para asignar el agua que los medios administrativos de regulación (zonas de 
veda, ${ }^{5}$ zonas reglamentadas, etc.). Los mercados de agua pueden ser formales o informales, por lo general funcionan informalmente en zonas de alta escasez, sobre todo cuando los gobiernos fallan en responder rápidamente a los cambios de demanda de agua (Kemper et al., 2003). Para que los mercados formales de agua sean efectivos son necesarios derechos de uso de agua, comprables y vendibles. Por esta razón, la aplicabilidad general de mercados formales de agua en países en desarrollo ha sido cuestionada, ya que requieren el establecimiento previo de instituciones que disminuyan los costos de transacción y la información asimétrica de los intercambios potenciales (Kemper et al., 2003).

El criterio principal para evaluar el desempeño de estos tres mecanismos es la eficiencia; es decir, que se maximice el beneficio neto total entre los diferentes usos posibles, que puede ser generado con las tecnologías existentes y los volúmenes disponibles y que también incluya los costos de instrumentación (Tsur, et al., 2004). El criterio de eficiencia se refiere, únicamente, a los beneficios totales que pueden ser generados, no a la manera en que dichos beneficios se distribuyen entre los usuarios del agua.

En ausencia de impuestos u otras distorsiones, una asignación que maximiza los beneficios netos totales es llamado Pareto eficiente o primer mejor, ya que, al moverse hacia esa asignación, los involucrados quedan mejor o igual que en la situación anterior. Cuando existen distorsiones, sean de información, institucionales, políticas u otras, la asignación es denominada segunda mejor, ya que al moverse hacia la nueva asignación habrá ganadores y perdedores.

Ahora bien, la provisión de servicios de agua presenta complejidades que hacen necesaria algún tipo de intervención pública. Por ejemplo, i) la mayoría de las inversiones en infraestructura hidroagrícola requieren

\footnotetext{
${ }^{5}$ La LAN establece que las aguas del subsuelo podrán ser libremente alumbradas excepto cuando el Ejecutivo Federal establezca zonas reglamentadas para su extracción o zonas de veda o de reserva. El primer Decreto de Veda se publicó en 1948 en el acuífero de la Comarca Lagunera. En 2007 existían 145 decretos de veda que cubren 53\% del territorio nacional.
} 
grandes montos de capital y periodos largos, antes de que las recompensas sean visibles, lo que hace difícil atraer inversionistas privados; ii) la distribución de agua presenta economías de escala, que la convierten en un monopolio natural; iii) existe información incompleta para la autoridad central sobre oferta y demanda de agua y iv) existen externalidades positivas por la provisión de agua (Easter, Rosegrant y Dinar, 1998) y negativas por un consumo irracional. Lo anterior implica que, en general, nos encontramos en un mundo del segundo mejor.

Basados en Tsur et al. (2004) el cuadro 2 ilustra la eficiencia de los diferentes métodos para el establecimiento de precios del agua para riego agrícola. La medición volumétrica alcanza una asignación de primer mejor por su facilidad para controlar los cambios en la demanda de agua, aunque tiene una instrumentación complicada por la necesidad de disponer de información sobre el consumo de agua realizado por cada usuario. Los mercados de agua, por su parte, también generan asignaciones de primer mejor en términos de eficiencia, aunque resulta difícil su funcionamiento si no existen instituciones preestablecidas (i.e. derechos de propiedad y mecanismos de cumplimiento y sanción de los contratos). Las tarifas en bloques y en dos partes requieren dispositivos de medición volumétrica y son aplicadas principalmente en el uso urbano de agua.

Así, por su facilidad de instrumentación, el método de establecimiento de precios más comúnmente utilizado en las zonas agrícolas es la medición no volumétrica, por lo general basada en el área cultivada. Sin embargo, dicho método no alcanza una asignación eficiente, dado que la extensión de área cultivada no se encuentra, necesariamente, vinculada al consumo de agua y, por lo tanto, no tiene tanta capacidad de controlar las variaciones en la demanda de agua.

Dado que los costos de instrumentación asociados con cada esquema de establecimiento de precios varían ampliamente de región a región por las variaciones en el clima, demografía, estructura social, derechos del agua, infraestructura del agua, aspectos históricos y condiciones económicas generales; los beneficios netos asociados con cada método también variarán de región a región. 
Cuadro 2

Propiedades de los métodos de establecimiento de precio del agua

\begin{tabular}{|c|c|c|c|c|}
\hline $\begin{array}{c}\text { Esquema } \\
\text { de precio }\end{array}$ & Instrumentación & $\begin{array}{l}\text { Eficiencia } \\
\text { alcanzada }^{6}\end{array}$ & $\begin{array}{l}\text { Horizonte de } \\
\text { tiempo de } \\
\text { eficiencia }^{7}\end{array}$ & $\begin{array}{c}\text { Capacidad de } \\
\text { controlar la } \\
\text { demanda }\end{array}$ \\
\hline Volumétrico & Complicada & $\begin{array}{l}\text { Primer } \\
\text { mejor }\end{array}$ & Corto plazo & Fácil \\
\hline Producto & Relativamente fácil & $\begin{array}{l}\text { Segundo } \\
\text { mejor }\end{array}$ & Corto plazo & Relativamente fácil \\
\hline Insumo & Fácil & $\begin{array}{l}\text { Segundo } \\
\text { mejor }\end{array}$ & Corto plazo & Relativamente fácil \\
\hline Por área & La más fácil & Ninguna & $\mathrm{N} / \mathrm{A}$ & Difícil \\
\hline $\begin{array}{l}\text { Tarifas en } \\
\text { bloque }\end{array}$ & $\begin{array}{c}\text { Relativamente } \\
\text { complicado }\end{array}$ & $\begin{array}{l}\text { Primer } \\
\text { mejor }\end{array}$ & Corto plazo & Relativamente fácil \\
\hline Dos partes & $\begin{array}{l}\text { Relativamente } \\
\text { complicado }\end{array}$ & $\begin{array}{l}\text { Primer } \\
\text { mejor }\end{array}$ & Largo plazo & Relativamente fácil \\
\hline $\begin{array}{c}\text { Mercados } \\
\text { de agua }\end{array}$ & $\begin{array}{l}\text { Difícil sin institucio- } \\
\text { nes pre-establecidas }\end{array}$ & $\begin{array}{l}\text { Primer } \\
\text { mejor }\end{array}$ & Corto plazo & $\begin{array}{l}\text { Fácil si existen insti- } \\
\text { tuciones que funcio- } \\
\text { nen adecuadamente }\end{array}$ \\
\hline
\end{tabular}

Fuente: Tsur et al. (2004)

\section{CAMBIO INSTITUCIONAL HACIA LA ADMINISTRACIÓN DE LA DEMANDA}

Para poder transitar de un esquema de administración de la oferta a uno de administración de la demanda vía precios se requiere que los costos de transacción del cambio institucional sean menores que el costo de oportunidad de no actuar y mantener el estado actual (Saleth y Dinar, 2004). En este sentido, los esfuerzos de reforma dirigidos al establecimiento de precios

\footnotetext{
${ }^{6}$ La eficiencia en la asignación definida como primer mejor no toma en cuenta la existencia de costos de instrumentación (i.e. administración, operación, acopio de información, etc.) o información incompleta (i.e. agua no medida). La segunda mejor sí toma en cuenta estos factores. ${ }^{7}$ La eficiencia de corto plazo ignora los costos fijos, en tanto que la eficiencia de largo plazo los contabiliza.
} 
"correctos" del agua, con frecuencia se originan de crisis financieras, bajo porcentaje de recuperación de costos, deterioro de la infraestructura, incremento en la demanda de agua y reformas macroeconómicas relacionadas (Tsur et al., 2004).

Existen varias dificultades para este cambio de paradigma. Entre ellas se encuentran la dificultad política de promulgación de leyes y creación de políticas e incentivos orientados a la asignación eficiente, así como la construcción de la estructura organizacional necesaria para traducir las leyes en práctica dentro de organizaciones con experiencia y tradición en el desarrollo de nuevas fuentes de abastecimiento del agua (ampliación de la oferta), y no de administración de la demanda.

De esta manera, el desarrollo de nuevas habilidades e información en las viejas administraciones del agua y la creación de organizaciones intersectoriales e interregionales adicionales resulta ser crítico para alcanzar los retos de un paradigma de administración de la demanda. A diferencia de la era del desarrollo de fuentes de abastecimiento, caracterizadas por el exceso de burocracia y el dominio de consideraciones políticas y de ingeniería hidráulica, la era de la asignación por el lado de la demanda requiere de: información económica y ecológica que disminuya las asimetrías de información e internalice las externalidades negativas, organizaciones de gobernanza que garanticen el cumplimiento y sanción de los derechos de propiedad y bajos costos de transacción e instrumentación para realizar los intercambios y alcanzar primeras o segundas mejor asignaciones.

Este cambio institucional sucede de manera natural al evolucionar las instituciones con las condiciones cambiantes y necesidades sociales. Sin embargo, el resultado final de tal evolución está definido por complicados procesos políticos en los que intervienen distintos grupos e intereses. Si los grupos de presión buscadores de rentas logran obstaculizar el proceso de reformas pueden persistir instituciones que generan asignaciones subóptimas. No obstante, con el incremento de la escasez del agua y del déficit fiscal las ganancias de los buscadores de rentas pueden disminuir y aumentar los costos sociales de mantener políticas inapropiadas en el sector hídrico, de modo que se generen las condiciones para el cambio. 
En México, si bien desde la promulgación de la Ley de aguas nacionales Conagua se ha propuesto utilizar la administración de las concesiones para lograr el objetivo de uso eficiente del recurso en el sector agrícola, a partir de la asignación de volúmenes concesionados; la vigilancia de que se extraiga hasta el volumen establecido, la instalación de medidores para monitorear la extracción de agua, el cobro de las cantidades de agua consumidas por encima de lo permitido y ser el intermediario para la transmisión de derechos (volúmenes) de agua entre los diferentes agentes económicos, según indican los análisis existen serias fallas en la aplicación de dichos mecanismos regulatorios de asignación del agua para uso agrícola (Shah et al., 2000).

Por un lado, los derechos de propiedad para el uso del agua establecidos a través de concesiones sobre la cantidad que es posible extraer han sido determinados inadecuadamente y existe un insuficiente monitoreo de las concesiones, ya que, tratándose de un recurso de acceso común como el agua subterránea, no existe exclusividad en el disfrute del recurso y tampoco indivisibilidad en su consumo. Los usuarios tienden a no tener incentivos para conservar el recurso, en virtud de que cada unidad que ellos dejen de consumir o preservar para un futuro incierto o de escasez es consumida por otros que tienen libre acceso a la explotación del recurso. Para el caso del agua subterránea, dado que la misma tabla está disponible para muchos usuarios, cada usuario inflige una externalidad a los otros, por lo que, sin derechos de propiedad bien establecidos, monitoreados y sancionados, no existen incentivos para la conservación del recurso (Zilberman y Schoengold, 2007).

Además, aunque en teoría un organismo gubernamental omnisciente podría estructurar un sistema óptimo de asignación de concesiones, en la práctica los costos de transacción son demasiado altos para que la burocracia obtenga información suficiente y auténtica sobre la medición de uso del recurso por parte de cada usuario (Roemer, 2007).

Por otro lado, Shah (2008) encontró que en México los volúmenes de agua concesionados están basados en una combinación del rendimiento del pozo y la extensión de la propiedad del usuario, por lo que 
las concesiones de agua subterránea solamente regularizan el estado actual y no tienen el objetivo de alinear la disponibilidad hidrológica de agua con los volúmenes otorgados en los títulos. Por su parte, la Auditoría Superior de la Federación (el máximo órgano de fiscalización del país) encontró en 2009 que Conagua había entregado títulos de concesión sin tomar en cuenta la disponibilidad hidrológica del acuífero, que la mayoría de los acuíferos aún no contaban con estudios de disponibilidad de agua publicados o actualizados y que no se estaba vigilando que los usuarios tuvieran instalado un medidor volumétrico para la extracción de agua.

Por otra parte, Conagua también intenta hacer uso de instrumentos participativos para administrar el uso del agua subterránea y disminuir los costos de hacer cumplir la LAN. Se han realizado esfuerzos por incorporar las necesidades de los usuarios y los acuerdos locales en la toma de decisiones en la administración del agua, a través de los Comités Técnicos de Aguas Subterráneas (Cotas) como órganos de representación de los usuarios de aguas nacionales de los acuíferos. Sin embargo, las expectativas que la autoridad del agua ha puesto sobre los mecanismos de gobernanza local son muy elevadas y no se encuentran necesariamente alineadas con el resto de las políticas centralizadas. Solamente 1 de cada 10 agricultores ubicados en los acuíferos sobreexplotados analizados conocía qué es el Cotas, como organismo colectivo coadyuvante para las decisiones de administración comunitaria del agua subterránea en México (INE-UACH, 2010). En todo caso, estas organizaciones de usuarios, en el corto plazo, no podrán reemplazar las tareas que por ley les corresponde realizar a las agencias burocráticas.

Como puede verse, los mecanismos utilizados actualmente no son los más efectivos para propiciar un uso eficiente del agua. Sin embargo, las instituciones formales vigentes le dan a Conagua los instrumentos y la flexibilidad para moverse hacia una administración de la demanda mediante mecanismos de asignación de precios. 


\subsection{Mecanismos de precios de medición volumétrica}

Los derechos que se deben pagar por la explotación, uso o aprovechamiento de aguas nacionales están establecidos en el artículo 223 de la Ley Federal de Derechos (LFD). Además, de acuerdo con la Ley de aguas nacionales, los agricultores deben contar con un medidor que registre su consumo de agua. El cuadro 3 señala los usos específicos y las cuotas que se han aplicado por los diferentes usos del agua, a saber, el régimen general, el agua potable, agropecuario, balnearios y centros recreativos, generación hidroeléctrica y acuacultura. El uso agropecuario tiene cuotas diferenciadas de acuerdo con el consumo observado, si estuvo por debajo o por encima de la concesión.

\section{Cuadro 3}

Cuotas por explotación, uso o aprovechamiento de aguas nacionales según zonas de disponibilidad, 2002 y 2008 (pesos por metro cúbico, pesos corrientes)

\begin{tabular}{|c|c|c|c|c|c|c|c|c|c|c|}
\hline \multirow{2}{*}{ Uso } & \multirow{2}{*}{ Año } & \multicolumn{9}{|c|}{ Zona de disponibilidad } \\
\hline & & 1 & 2 & 3 & 4 & 5 & 6 & 7 & 8 & 9 \\
\hline \multirow{2}{*}{$\begin{array}{c}\text { Régimen } \\
\text { general }\end{array}$} & 2008 & 16.5665 & 13.2527 & 11.0438 & 9.1113 & 7.1783 & 6.4876 & 4.8831 & 1.7349 & 1.3002 \\
\hline & 2002 & 13.3885 & 10.7105 & 8.9253 & 7.3636 & 5.8014 & 5.2431 & 3.9466 & 1.4023 & 1.0509 \\
\hline \multirow{2}{*}{$\begin{array}{c}\text { Agua potable, } \\
\text { consumo } \\
\text { mayor a } 300 \text { I/ } \\
\text { hab.-día }\end{array}$} & 2008 & 0.65637 & 0.65637 & 0.65637 & 0.65637 & 0.65637 & 0.65637 & 0.30566 & 0.15265 & 0.03799 \\
\hline & 2002 & 0.2652 & 0.2652 & 0.2652 & 0.2652 & 0.2652 & 0.2652 & 0.1235 & 0.0062 & 0.0031 \\
\hline \multirow{2}{*}{$\begin{array}{c}\text { Agua potable, } \\
\text { consumo igual } \\
\text { o inferior a } 300 \\
\text { l/hab.-día }\end{array}$} & 2008 & 0.32819 & 0.32819 & 0.32819 & 0.32819 & 0.32819 & 0.32819 & 0.15283 & 0.07632 & 0.03799 \\
\hline & 2002 & 0.2652 & 0.2652 & 0.2652 & 0.2652 & 0.2652 & 0.2652 & 0.1235 & 0.0062 & 0.0031 \\
\hline \multirow{2}{*}{$\begin{array}{l}\text { Agropecuario, } \\
\text { sin exceder } \\
\text { concesión }\end{array}$} & 2008 & 0.00 & 0.00 & 0.00 & 0.00 & 0.00 & 0.00 & 0.00 & 0.00 & 0.00 \\
\hline & 2002 & 0.00 & 0.00 & 0.00 & 0.00 & 0.00 & 0.00 & 0.00 & 0.00 & 0.00 \\
\hline \multirow{2}{*}{$\begin{array}{c}\text { Agropecuario, } \\
\text { por cada m3 } \\
\text { que exceda } \\
\text { el volumen } \\
\text { concesionado }\end{array}$} & 2008 & 0.0001173 & 0.0001173 & 0.0001173 & 0.0001173 & 0.0001173 & 0.0001173 & 0.0001173 & 0.0001173 & 0.0001173 \\
\hline & 2002 & 0.00 & 0.00 & 0.00 & 0.00 & 0.00 & 0.00 & 0.00 & 0.00 & 0.00 \\
\hline \multirow{2}{*}{$\begin{array}{c}\text { Balnearios y } \\
\text { centros recrea- } \\
\text { tivos } \\
\end{array}$} & 2008 & 0.0094231 & 0.0094231 & 0.0094231 & 0.0094231 & 0.0094231 & 0.0094231 & 0.0046424 & 0.0021855 & 0.0010392 \\
\hline & 2002 & 0.0076 & 0.0076 & 0.0076 & 0.0076 & 0.0076 & 0.0076 & 0.0038 & 0.0018 & 0.0008 \\
\hline \multirow{2}{*}{$\begin{array}{c}\text { Generación } \\
\text { hidroeléctrica }\end{array}$} & 2008 & 0.0034824 & 0.0034824 & 0.0034824 & 0.0034824 & 0.0034824 & 0.0034824 & 0.0034824 & 0.0034824 & 0.0034824 \\
\hline & 2002 & 0.0028 & 0.0028 & 0.0028 & 0.0028 & 0.0028 & 0.0028 & 0.0028 & 0.0028 & 0.0028 \\
\hline \multirow{2}{*}{ Acuacultura } & 2008 & 0.002705 & 0.002705 & 0.002705 & 0.002705 & 0.002705 & 0.002705 & 0.0013321 & 0.0006264 & 0.0002975 \\
\hline & 2002 & 0.0022 & 0.0022 & 0.0022 & 0.0022 & 0.0022 & 0.0022 & 0.0011 & 0.0005 & 0.0002 \\
\hline
\end{tabular}

Fuente: Elaboración propia con información de la Ley Federal de Derechos, últimas reformas publicadas en el DOF, el 13 de noviembre de 2008, y de Conagua, 2003 
Como puede observarse en el cuadro 3, la LFD exenta a los agricultores de pago, salvo aquellos volúmenes consumidos por encima de lo establecido en el título de concesión; y, aun para ellos, la cuota es la más baja de todos los usos contemplados en la Ley. Además, existen problemas de cumplimiento de ley en materia de instalación de medidores, por lo que, en muchos casos, no existe información sobre el consumo de agua de los productores (Auditoría Superior de la Federación, 2009).

\subsection{Mecanismos de precios de medición no volumétrica}

A pesar de que no existe un precio directo por el consumo de agua dentro de la concesión para uso agropecuario, los agricultores pagan el precio indirecto del agua a través de la energía eléctrica que utilizan para bombear y rebombear agua del subsuelo hacia sus parcelas.

La Secretaría de Hacienda y Crédito Público (SHCP) establece las tarifas eléctricas para bombeo agrícola y las publica en el Diario Oficial de la Federación (DOF). Sin embargo, en 2003, a raíz del Acuerdo Nacional para el Campo, se establecieron las llamadas tarifas eléctricas de estímulo $9 \mathrm{CU}$ y $9 \mathrm{~N}$ en la Ley de Energía para el Campo (LEC) para mantener a los agricultores mexicanos en condiciones de competitividad respecto a los principales socios comerciales del país. Como puede observarse en el cuadro 4, en 2009 las tarifas eléctricas para bombeo agrícola que pagaron los agricultores mexicanos representó solo una cuarta parte de lo que pagaron los productores agrícolas de Estados Unidos. Entre 2003 y 2009 las tarifas de estímulo aumentaron 40\%, mientras que las tarifas normales (9 y 9M) se incrementaron en casi 300\%. Por este motivo, Shah (2008) menciona que una de las principales razones por las que los agricultores buscan tener una concesión de agua, no es para evitar las multas y sanciones de Conagua, sino para tener acceso a las tarifas de estímulo de electricidad para bombeo y rebombeo agrícola. ${ }^{8}$

\footnotetext{
${ }^{8}$ Es condición necesaria para acceder a los subsidios a la electricidad contar con una concesión de agua vigente.
} 


\section{Cuadro 4}

Comparación de tarifas agrícolas en México y Estados Unidos

(pesos por kwh)

\begin{tabular}{|l|c|c|c|c|}
\hline \multirow{2}{*}{ Año } & \multicolumn{3}{|c|}{ México } & Estados Unidos \\
\cline { 2 - 3 } & Tarifas de estímulo & Tarifas normales \\
& $9 \mathrm{CU}$ & $9 \mathrm{~N}$ & ${\text { T } 9)^{2 /}}^{2 /}$ & Tarifas agrícolas $^{3 /}$ \\
\hline 2003 & 0.30 & 0.15 & 0.41 & 0.72 \\
\hline 2004 & 0.32 & 0.16 & 0.52 & 0.83 \\
\hline 2005 & 0.34 & 0.17 & 0.66 & 0.83 \\
\hline 2006 & 0.36 & 0.18 & 0.84 & 0.94 \\
\hline 2007 & 0.38 & 0.19 & 0.98 & 0.96 \\
\hline 2008 & 0.40 & 0.20 & 1.31 & 1.42 \\
\hline 2009 & 0.42 & 0.21 & 1.63 & 1.37 \\
\hline
\end{tabular}

Fuente:1/ DOF, 7 de enero y 8 de agosto de 2003 y sus modificaciones, 2/DOF, 7-I-2003 y sus modificaciones, 3 / Energy Information Agency (EIA)

Sagarpa otorga a cada agricultor una cuota energética máxima que puede pagarse con las tarifas de estímulo. El consumo de energía eléctrica excedente se paga con las tarifas normales 9 y $9 \mathrm{M}$ según el rango de energía consumida (ver gráfica A1 del anexo). Según el esquema actual, las tarifas agrícolas $9 \mathrm{CU}$ y $9 \mathrm{~N}$ tienen un subsidio de $83 \%$, y se incrementan dos centavos al año; en tanto que las tarifas 9 y $9 \mathrm{M}$ (que difieren entre ellas por el nivel de tensión de energía) están menos subsidiadas y tienen aumento mensual de dos centavos. La cuota energética es otorgada de acuerdo con una fórmula, cuya variable principal son los caballos de fuerza del equipo de bombeo que tiene el agricultor, ${ }^{9} \mathrm{y}$ un indicador de la cantidad de agua que requiere el agricultor para satisfacer sus necesidades de riego, con base en las características de su unidad de producción y la profundidad del pozo, entre otros aspectos. Sin embargo, actualmente la cuota energética sobreestima el verdadero consumo de energía eléctrica de los agricultores, ya que $93 \%$ de los agricultores consumen por debajo de ella, lo que garantiza que los agricultores, casi siempre, paguen energía

\footnotetext{
${ }^{9}$ Cuota energética $(\mathrm{CE})=(\mathrm{HP} \times 0.746 \times 365 \times 24) \times 0.75+438$; Donde HP = capacidad del motor de la bomba.
} 
eléctrica a tarifas de estímulo. Adicionalmente, si su cuota energética no es suficiente, el agricultor sólo requiere solicitar un aumento a Sagarpa.

Por esta razón, los mecanismos de precios a través de mediciones no volumétricas, tampoco han incentivado un uso más eficiente del recurso y distan de poner a México en una situación de administración de la demanda.

\subsection{Mecanismos de precios a través de mercados de agua}

En México los intercambios en mercados de agua pueden desarrollarse plenamente cuando el acuífero esté declarado como zona de veda, ya que, al existir un límite de extracción, Conagua no puede expedir más títulos de concesión que los ya vigentes en el mercado, y, dado que los usuarios deben poseer un título de concesión, la única vía para regularizarse es intercambiar derechos de agua.

En los últimos años las transmisiones de derechos han ido en aumento, especialmente en las zonas en las que existe un mayor grado de presión del recurso hídrico. En 2001 se registraron 672 transacciones y en 2008 se incrementaron a 2821 , la mayoría correspondientes a aguas subterráneas y a uso final agrícola (Aguilar y Canizales, 2008).

Destaca que 90\% del volumen total transmitido por el sector agrícola fue intercambiado entre los propios usuarios agrícolas, lo que puede indicar que dicho mecanismo asigna el recurso hacia aquellos agricultores que más lo valoran, es decir, el agua estaría llegando hacia los agricultores que poseen un mayor costo de oportunidad de no tenerla, que a los agricultores que actualmente tienen el derecho de usarla (Canizales, 2009).

La transmisión de derechos de agua se ve fortalecida con el adecuado funcionamiento de los mecanismos de cumplimiento y sanción de los contratos, así como con el establecimiento de organizaciones que disminuyan las asimetrías de información y los costos de transacción de encontrar a oferentes y demandantes, es decir, instituciones que faciliten la obtención de acuerdos, elaboración y cumplimiento de contratos. 
En ese sentido, el artículo 37 Bis de la Ley de Aguas Nacionales prevee el establecimiento definitivo o temporal de bancos de agua que proporcionen información confiable, certera y oportuna sobre las ofertas y demandas de agua existentes en una región hidrológica específica. Hasta septiembre de 2009 se habían creado tres bancos de agua para apoyar la gestión de las transmisiones de derechos de agua. ${ }^{10}$ Sin embargo, existen en México 145 decretos de veda, por lo que son necesarias muchas más instancias para, efectivamente, disminuir los costos de transacción y desarrollar el potencial del mercado de transmisiones de derechos.

Un problema para el desarrollo de los mercados de agua en México es que, al ser este recurso propiedad de la nación (artículo 27 constitucional), en sentido estricto no se puede comercializar; por ello Conagua solo registra oficialmente las cantidades intercambiadas y no tiene control sobre la información de los precios al que los agricultores compran y venden los derechos de agua ${ }^{11}$ en la práctica. Los agricultores acuerdan por fuera el precio al que se realizará la transmisión de derechos y luego, en su caso, acuden a la Conagua a registrar el intercambio. Al no llevarse el registro oficial de los precios a los que se transfieren los derechos de agua se pierde información valiosa sobre la escasez relativa del recurso en el acuífero y los bancos de agua tampoco pueden cumplir su función de disminuir los costos de transacción entre los oferentes y demandantes.

De superarse esos retos institucionales se tiene un gran potencial en el desarrollo de mercados de agua para asignar el recurso a sus mejores usos, ya que, si bien 70\% de los agricultores sabe que la Ley de Aguas Nacionales le permite intercambiar derechos de agua hacia otros agricultores o actividades económicas alternativas del acuífero, solamente 25\% manifestó haber participado en alguna transacción (compra, venta, renta) de este tipo (INE-UACH, 2010).

\footnotetext{
${ }^{10}$ Los tres bancos de agua se encuentran en las cuencas centrales del norte (Región Lagunera, sede en Coahuila), cuenca Lerma-Santiago-Pacífico (abarca nueve estados, con sede en Jalisco) y el último en la cuenca Río Bravo (Nuevo León).

${ }^{11} \mathrm{http}$ ://www.jornada.unam.mx/2009/07/09/index.php?section=sociedad\&article=044 n1soc
} 


\section{ANÁLISIS DE ECONOMÍA POLÍTICA DE LOS MECANISMOS DE ASIGNACIÓN DEL AGUA EN MÉXICO}

Para entender por qué no se usan plenamente los mecanismos existentes para la asignación de precios al agua en México es necesario hacer un análisis de economía política, es decir, cómo afectan las fuerzas políticas la selección de instrumentos en términos de conflictos distributivos e instituciones políticas.

En las siguientes secciones utilizaremos un modelo sencillo de equilibrio parcial de mercado de agua subterránea para entender tales dinámicas, y cómo afectan la asignación final del recurso. Esto es con la finalidad de poder proponer arreglos institucionales que lleven a una asignación más eficiente. El primer paso para el análisis es entender cómo sería una asignación eficiente y cómo los arreglos institucionales vigentes llevan, o no, a esa asignación.

\subsection{Análisis de equilibrio económico sin asignación intertemporal}

La Ley Federal de Derechos exenta a los agricultores del pago de agua que consumen, siempre que sea dentro de su concesión. Es decir, no pagan el costo directo de extracción (CME), ni de distribución del recurso (CMD), ni las externalidades a generaciones presentes (CMA), ni el costo de oportunidad de las generaciones futuras (CMF). Así también, el precio indirecto de extracción (CMEi) (medido por el costo de la energía eléctrica utilizada para bombear agua del subsuelo) se encuentra fuertemente subsidiado por las tarifas de estímulo (CMEs) establecidas en la Ley de Energía para el Campo.

Como se observa en la gráfica 1, en un acuífero en equilibrio o con margen de extracción, el equilibrio socialmente óptimo estaría dado por el punto A, donde la curva de demanda (BMgs) cruza la recta CMEi + CMD + CMA + CMF. Lo que da como resultado un precio óptimo $\mathrm{P}^{*}$, y una cantidad extraída óptima $\mathrm{m}^{*}{ }^{12}$ Sin embargo, al subsidiarse el costo de extracción a través de la tarifa eléctrica y no incluirse el costo de almace-

\footnotetext{
${ }^{12}$ Por cuestiones de simplicidad asumimos que $\mathrm{m}^{*}$ es también igual al volumen de extracción sustentable, es decir, que iguala el volumen de precipitación anual y recarga del
} 
namiento para los usuarios futuros, ni el costo de la externalidad ambiental (intrusión salina, contaminación con arsénico, etc.), ni el costo de distribución; lo que tenemos es un resultado subóptimo en el punto $\mathrm{D}$, con un precio Ps y una cantidad ms, donde $\mathrm{Ps}<\mathrm{P}^{*} \mathrm{y} \mathrm{ms}>\mathrm{m}^{*}$. El exceso de consumo de agua es representado por la distancia $\mathrm{ms}-\mathrm{m}^{*} \mathrm{y}$ el diferencial entre $\mathrm{P}^{*} \mathrm{y}$ Ps mide tanto la falla de mercado como la falla de política del arreglo institucional vigente (Barde y Honkatukia, 2003). Algunos autores (Saleth y Dinar, 2004) asocian ese diferencial de precios al resultado del cabildeo y presión política de los grupos de interés que se apropian de las rentas creadas ${ }^{13}$ por una decisión de gobierno. ${ }^{14} \mathrm{El}$ área AJD representa la pérdida neta del bienestar total de la sociedad, que genera el mecanismo de precios directos e indirectos del agua previstos en el arreglo institucional vigente. ${ }^{15}$

Incluir en el precio cualquiera de estos costos o disminuir el subsidio a la electricidad nos acercará, todo lo demás constante, al equilibrio socialmente óptimo $\mathrm{m}^{*}$. Para llegar al equilibrio óptimo los tomadores de decisiones pueden elegir entre controlar la cantidad de extracción de agua al establecer un precio mínimo igual a P2 o vigilar que la extracción del agua no sobrepase $\mathrm{m}^{*}$ (por ejemplo, monitorear que las concesiones se cumplan). En presencia de información perfecta, ausencia de costos de

acuífero (flujo), y que niveles de consumo superiores a $\mathrm{m}^{*}$ ya empiezan a utilizar la reserva de agua que fue acumulada durante décadas, por lo que consumos superiores a $\mathrm{m}^{*}$ disminuyen el acervo de agua disponible para los usuarios del futuro.

${ }^{13}$ Brubaker (1997) hace una diferencia muy importante entre la creación y la distribución de una renta. La aprobación de una legislación que hace posible la emergencia de una renta en un mercado particular es un tipo de bien de consumo colectivo, ya que múltiples agentes tienen acceso potencial en la repartición de la renta una vez que ésta ya fue creada. Si seguimos el argumento, el problema del free-rider limitará la disposición de los agentes a invertir en cabildeo y otras actividades para conseguir el beneficio de la acción del gobierno. Una vez creada la renta, sin embargo, la renta es divisible, lo cual sugiere que el desperdicio social asociado con la búsqueda de rentas es, probablemente, más serio cuando se trata de la distribución de rentas.

${ }^{14}$ La presión de los grupos de interés puede ser aún más elevada si el subsidio se encuentra concentrado en los agricultores más ricos, con mayores posibilidades de movilización, conexión y cabildeo con los tomadores de decisiones del Poder Ejecutivo y Legislativo. De acuerdo con los datos de consumo de la CFE para 2008 el índice de Gini es 0.69, donde o es igualdad absoluta y 1 refleja una desigualdad total en la distribución del subsidio.

${ }^{15}$ La pérdida neta del bienestar social total podría ser infinita si se considera una curva de beneficio marginal social total asintótica al eje x y y. 
transacción y de incertidumbre, ambos instrumentos de política son equivalentes (Weitzman, 1974). No obstante, tal y como señalan Tsur et al. (2004) para el caso del agua, en la práctica los costos de transacción son enormes y la información de la que disponen las autoridades del agua es a menudo incompleta. Esta situación se agrava con la incertidumbre asociada a la alta dispersión en los resultados de las proyecciones de las variaciones en los patrones y localización de la precipitación en los modelos climáticos existentes ${ }^{16}$ que podrían exacerbar la escasez hidrológica y económica presente.

\section{Gráfica 1}

Análisis estático del precio y extracción óptima del agua en un acuífero en equilibrio hidrológico

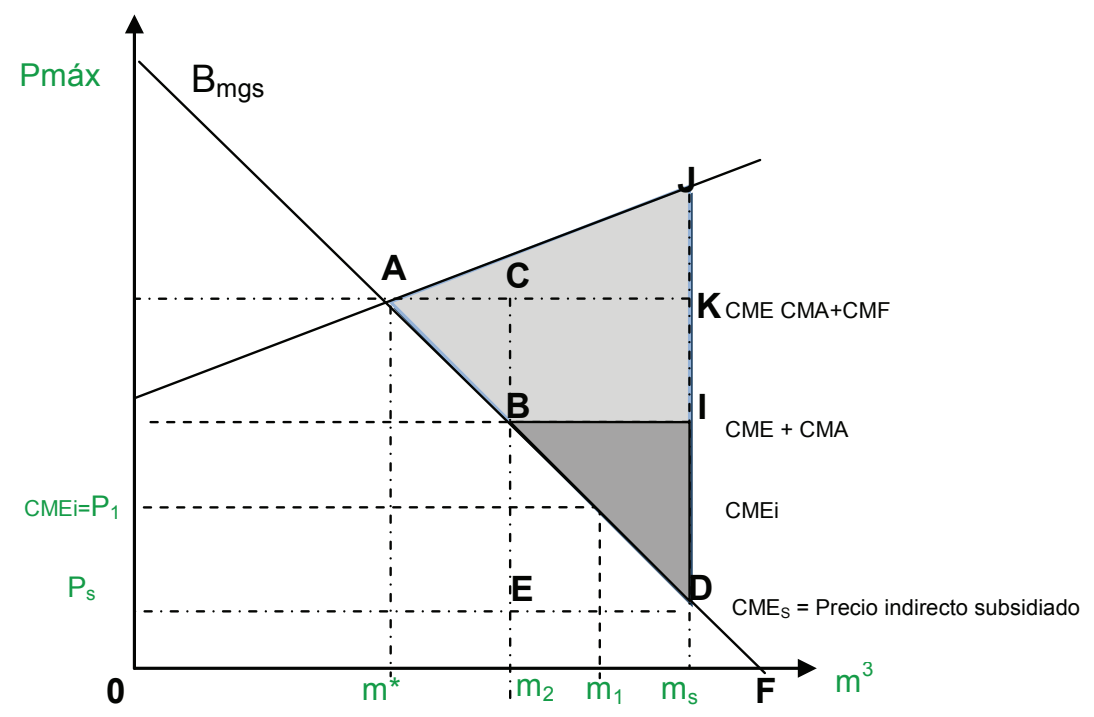

Donde:

- CMD: Costo marginal de distribución;

- CME: Costo marginal de extracción;

- $\mathrm{CME}_{\mathrm{s}}$ : Costo marginal de extracción con subsidio;

- CMA: Costo marginal ambiental (externalidad);

- CMF: Costo marginal de almacenamiento para las generaciones futuas;

- $\mathrm{B}_{\mathrm{mg}}$ : Beneficio marginal $=$ Demanda.

Fuente: Elaboración propia, adaptado de Zilberman y Schoengold (2007) y OCDE (2003)

${ }^{16}$ Para mayor información ver Galindo (2008). 
Si se estableciera un precio mínimo igual a $\mathrm{P}_{2}$ a través del aumento de la tarifa eléctrica, el excedente de los agricultores disminuiría en el área $\mathrm{P}^{*} \mathrm{AP} \max$, en tanto que el gobierno federal recaudaría adicionalmente BDE, que podría convertirse en una transferencia desacoplada del consumo de energía ${ }^{17}$ que los agricultores utilizarían para inversión en sistemas de riego más eficientes y ahorradores de agua. Por otra parte, si se fijaran las cantidades de agua, se vigilara que nadie extrajera por encima de ese volumen y hubiera intercambios de agua sin un precio oficial de por medio (como ocurre en los mercados formales de derechos de agua en México), la recaudación del gobierno sería cero. Según se puede observar en la gráfica 1, ante un incremento en la tarifa eléctrica $\left(\mathrm{P}_{2}\right)$ la pérdida neta del bienestar social disminuye en la cuantía del área BID.

Aún con una política de desacoplamiento del subsidio a la tarifa eléctrica se incurriría en una pérdida neta representada por el área AJIB, ya que no existe una medida que, por sí sola, lleve al uso sustentable del agua entre los diferentes usos que demanda la sociedad, pues existen otros instrumentos de política pública que también tendrían que ser instrumentados en sectores diferentes al agrícola (i.e. avanzar hacia esquemas de tarifas y precios más eficientes en los hogares urbanos, disminuir las fugas en las tuberías de las ciudades y el cumplimiento de la ley de aguas nacionales, entre otros) para alcanzar un nivel de extracción óptima o sustentable.

La diferencia entre el excedente de los agricultores con el subsidio a la energía eléctrica, y sin él, es una medida indirecta de la presión que los grupos de interés pueden ejercer sobre el gobierno para instaurar o mantener

\footnotetext{
${ }^{17}$ De acuerdo con el ERS-USDA (2004) y Barde yHonkatukia (2003) existen subsidios que tienen una racionalidad política "buena", pero cuya incidencia puede tener efectos regresivos o ambientalmente dañinos, por lo que, en este caso, se recomienda desvincular (o desacoplar) los subsidios del uso de los insumos, de la producción o del consumo, para obtener ganancias económicas, sociales y ambientales. Estos subsidios desacoplados se pueden convertir en transferencias al ingreso, en montos fijos, que les permitiría tomar decisiones sobre su producción o consumo, con base en las señales que envíe un mercado sin distorsiones. Estos subsidios están clasificados dentro de la caja verde de la OMC, ya que causan mínimas distorsiones al comercio (Paquin, et al., s/f).
} 
un esquema de subsidios que les permita obtener mayores ganancias a costa de otros agricultores presentes y futuros, así como de otros usuarios de agua de las ciudades (doméstico, industrial, servicios).

La disposición de los beneficiarios potenciales a invertir en actividades de búsqueda de rentas durante la fase de la creación de la Ley de Energía para el Campo (origen de ese diferencial) vis a vis durante la fase de distribución de los subsidios que se derivan de ella, dependió del valor esperado de las rentas que obtendrían los agricultores una vez que ésta fuera ya creada. Dado que la asignación final prácticamente fue "asegurada” por parte de los agricultores ricos y legales, solo se requería el título de concesión para poder acceder al reparto de las mismas, los buscadores de rentas invirtieron mayores recursos durante la fase de creación que durante la fase de asignación o distribución, en donde sólo buscan cumplir los requisitos administrativos (Rivero y Nieto, 2011).

Por otro lado, ningún mecanismo intermedio de asignación de precios (i.e. $\mathrm{P}_{2}$ ), por más distorsionado que sea, puede superar las enormes ganancias que tienen los agricultores mediante un mecanismo de asignación vía cantidades, ya que, en dicha situación, los agricultores obtendrían un excedente oPmax F. Las ganancias se vuelven todavía mayores si el esquema funciona de manera ineficiente, es decir, sin derechos de propiedad adecuadamente establecidos, débil monitoreo, vigilancia y sanción de las concesiones y altos costos de transacción de los intercambios de derechos de agua.

Es por esta razón que los agricultores mexicanos ejercen enormes presiones por mantener el status quo mixto, que combina un mecanismo de precios y tarifas con subsidios altamente distorsionantes para el consumo de agua, con un mecanismo de asignación vía cantidades incapaz de alcanzar sus objetivos en el consumo eficiente y sustentable del agua.

En el caso de un acuífero con sobreexplotación extrema se hacen más inclinadas las curvas de costo marginal social total, ya que cada unidad adicional de agua consumida tiene un mayor costo de oportunidad para las generaciones futuras. La mayor inclinación de la curva de costo 
marginal total aumenta la brecha entre $\mathrm{m}^{*}-\mathrm{m}_{\mathrm{s}} \mathrm{y} \mathrm{P}^{*}-\mathrm{P}_{\mathrm{s}} \mathrm{y}$ también la pérdida neta del bienestar (AJD) resulta ser mucho mayor, debido a las ganancias en bienestar a las que renuncian las generaciones presentes y futuras por la existencia del subsidio.

De esta manera, los efectos de una reforma institucional -i.e. desacoplamiento del subsidio al precio indirecto de extracción de agua subterránea- serían mucho mayores en acuíferos sobreexplotados, la existencia por sí sola de esta pérdida de bienestar sería un factor importante que podrían utilizar los ganadores (otros usuarios de agua diferentes del agrícola) para impulsar la reforma (Saleth y Dinar, 2004).

Sin embargo, a juicio de Muñoz et al. (2007), existen diversos factores que podrían influir en que se mantenga el estado actual, en cuanto a la distribución de subsidios y exenciones en el sector agrícola, entre ellos se encuentran: 1) los beneficios de las transferencias gubernamentales que reciben los grupos de interés están concentrados en pocas personas, mientras que los costos están dispersos en toda la sociedad, 2) existe información asimétrica entre la población en general y los grupos de interés que proveen de información estratégica a través del cabildeo a los tomadores de decisiones, 3) algunos agricultores ofrecen su respaldo político a quien les otorga mayores transferencias, 4) los distritos electorales agrícolas están sobrerrepresentados en el Congreso, 5) la mayoría de las actividades agrícolas tienen un impacto en el medio ambiente, pero la organización de los grupos medioambientales se encuentra menos desarrollada que la de los grupos de interés agropecuario y 6) los costos de transacción de reformar los subsidios agrícolas son elevados.

La gráfica 1 se refiere a una primera mejor asignación, es decir, no considera la existencia de costos de transacción y supone la existencia de información completa. No obstante, en la realidad los costos de transacción (cumplimiento de contratos, monitoreo, medición, obtención de información, etc.) y costos políticos (obtención de acuerdos) asociados a la extracción del agua subterránea son positivos, por lo que las agencias gubernamentales se encontrarán sujetas a la presión de los buscadores de rentas, que tratan de mantener su situación de ganancias privadas. 
La literatura sugiere incorporar estos costos de transacción en los costos marginales, lo cual puede disminuir las ganancias en eficiencia de una reforma institucional (Dinar, 2000).

\subsection{Análisis de equilibrio económico con asignación intertemporal}

En la sección anterior analizamos la asignación óptima del agua subterránea al suponer que las cantidades extraídas y consumidas en el presente son independientes o no afectan las asignaciones óptimas en el futuro; lo cual sería cierto si el acervo de agua almacenada en los acuíferos permaneciera constante en el tiempo, lo que solo podría ocurrir si los flujos anuales de recarga fueran mayores o iguales a las cantidades extraídas y consumidas anualmente de los mismos.

Tsur et al. (2004) afirman que, si en un periodo la diferencia entre la recarga natural y la extracción es negativa, entonces el acervo de agua en el próximo periodo será más pequeño, y, si esta situación persistiera, eventualmente se acabaría. El impacto inmediato en el próximo periodo es que la extracción será más cara. En el largo plazo la extracción podría restringirse a no exceder la recarga natural o podría cesar completamente si el acervo del agua queda arruinado (i.e. intrusión salina o contaminación con arsénico). En tal situación, la extracción y consumo presente no se encuentran totalmente desvinculados del futuro.

Tietenberg (2006) también señala que, cuando la extracción excede la recarga en un acuífero, el recurso será eventualmente agotado, ya sea cuando el acervo se termine o cuando el costo marginal de extraer agua adicional llegue a ser demasiado elevado o prohibitivo; ya que en mercados de agua subterránea eficientes dicho costo se incrementaría a medida que el nivel del agua disminuyera.

Adicionalmente al costo marginal de extracción se debe incluir la no disponibilidad en el futuro de cualquier unidad de agua utilizada en el presente. En mercados de agua subterránea eficientes, si el costo marginal total, de extracción (e.g. energía eléctrica para bombeo agrícola) y 
de usuario, se incrementara a medida que la tabla de agua disminuye, el precio del agua se incrementaría proporcionalmente y el consumo disminuiría sobre el tiempo hasta detenerse por completo.

La gráfica 2 ilustra el caso en el que la extracción y el consumo de un recurso que se agota (e.g. agua subterránea cuya extracción excede la recarga) disminuyen a lo largo del tiempo, a medida que los costos marginales totales se incrementan, lo que refleja una mayor escasez del recurso. En este caso, existe una fuente alternativa de obtención de un recurso renovable (e.g. agua superficial) a un costo marginal constante. La extracción y el consumo del recurso que se agota terminan en el momento en el que el costo marginal total de obtener una unidad adicional del recurso es mayor al beneficio marginal de consumir esa unidad o al costo marginal constante de sustituirlo por un recurso renovable.

Aun en el supuesto de que sean conocidos los costos marginales de extracción y distribución y las externalidades ambientales, persistiría la incertidumbre del verdadero valor que los usuarios del futuro estarían dispuestos a pagar por el agua que podrían dejar de consumir, en caso de que las generaciones presentes estuvieran disminuyendo el acervo de agua disponible para el consumo futuro, es decir, el costo de oportunidad o costo marginal del usuario.

\section{Gráfica 2}

La asignación óptima intertemporal del agua subterránea

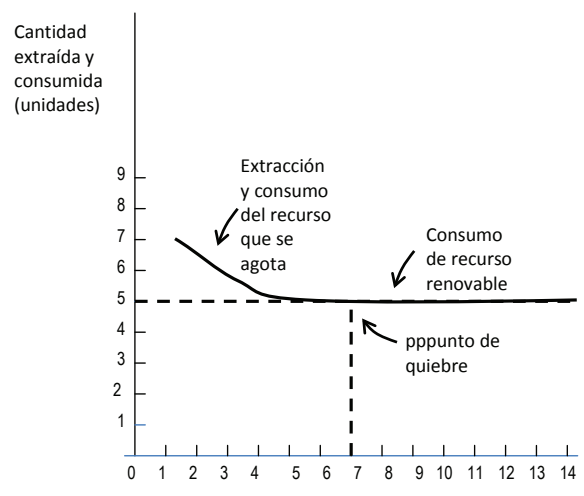

(a)

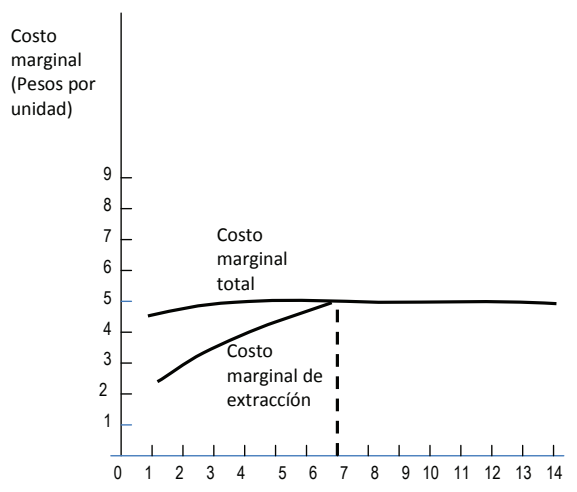

(b)

Fuente: Elaboración propia adaptada de Tietenberg (2006) 
$\mathrm{Al}$ abandono del supuesto de cero costos de transacción hay que sumarle, también, las implicaciones en la asignación óptima de incluir la presencia de incertidumbre en la oferta y demanda de agua subterránea en acuíferos sobreexplotados. Por el lado de la oferta existe incertidumbre cuando los patrones de precipitación, recarga y disponibilidad de agua son muy cambiantes, lo cual sucede en un contexto de cambio climático, en donde existe gran incertidumbre no solo de los signos de las variaciones de lluvia, sino de su magnitud, frecuencia y localización. Por otra parte, cuando un acuífero se encuentra en sobreexplotación extrema y los niveles de abatimiento hacen que se empiece a extraer agua de deficiente calidad, se puede desconocer con precisión la magnitud del costo de control del daño (e.g. costos de instalar y operar tecnologías que traten agua subterránea de deficiente calidad o contaminada con arsénico por los niveles de abatimiento) y/o el costo marginal del daño (costos adicionales a la sociedad por mayores gastos en salud por tomar agua contaminada). En presencia de incertidumbre, no siempre la administración de la demanda vía precios es el mejor mecanismo de asignación del agua entre sus diferentes usos.

Lo que sucede en la realidad es que, cuando existe un elevado nivel de incertidumbre e información incompleta, se desconocen la sensibilidad que tienen las curvas de costos y beneficios marginales totales en los puntos cercanos a la asignación óptima, lo que terminaría repercutiendo en la elección del mejor instrumento que maximiza las ganancias netas para la sociedad.

Tietenberg (2006) sugiere que en un contexto en el que la curva de costo marginal de control del daño sea muy plana (más elástica) y la curva de costo marginal del daño evitado sea muy inclinada, como en la gráfica 3, el uso de un mecanismo de control vía cantidades (i.e. prohibición de extracción de agua después de cierto nivel) sería un mejor instrumento de política que el controlar la asignación óptima vía precios, siempre y cuando existan derechos de propiedad bien definidos y un óptimo cumplimiento de la regulación. Pequeños cambios en la cantidad de asignación óptima generan enormes variaciones en el costo marginal de daño (i.e. mayores gastos en enfermedades por consumir agua con arsénico). En otras 
palabras, el costo de equivocarse y elegir un precio erróneo como mecanismo de asignación de agua subterránea, por ejemplo $\mathrm{P}_{\mathrm{c}}$, tiene mayores costos para la sociedad, por el daño marginal causado por variaciones en los niveles de extracción (y contaminación) óptima, en tanto que los costos de control para los contaminadores son, prácticamente, insensibles a las variaciones en los niveles de contaminación.

En la situación anterior, en la que el costo marginal de control es conocido pero el del daño es desconocido (o las proyecciones tienen un elevado grado de incertidumbre), es mejor establecer prohibiciones a la sociedad sobre las cantidades óptimas de extracción de agua sobre el nivel m*, porque unidades adicionales de consumo del recurso por encima de ese nivel generan un gran daño a la sociedad.

\section{Gráfica 3}

Caso 1: acuífero con sobreexplotación extrema en condiciones de incertidumbre

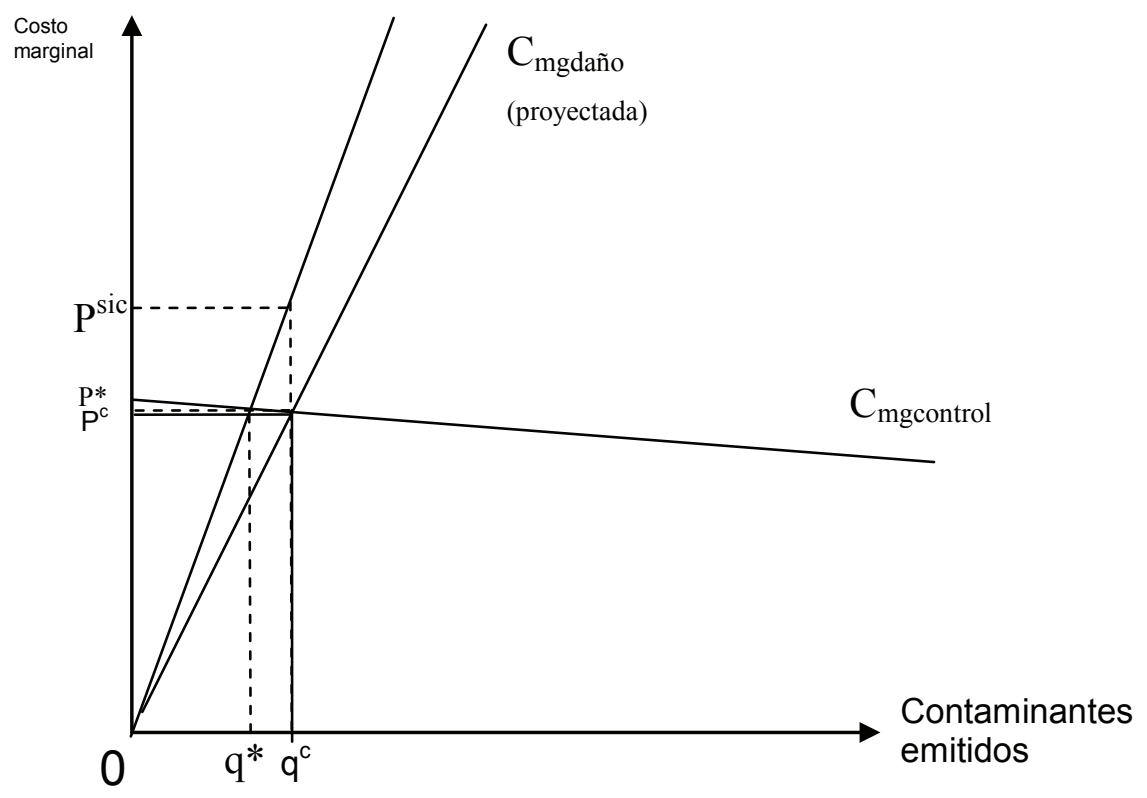

Fuente: Tietenberg (2006) 
Por otra parte, si la curva de costo marginal de control es desconocida y más inclinada (gráfica 4) y la curva de costo marginal del daño es conocida y casi plana, es decir, prácticamente insensible a las variaciones en la extracción del recurso, el uso de un mecanismo de precios para controlar la asignación óptima es el mejor instrumento de política, y habría que dejarles a los usuarios (contaminadores) la decisión de cuánto extraer, ya que el precio al que se enfrentan por nivel de extracción internalizaría el daño causado y, por lo tanto, buscarían de manera descentralizada la mejor tecnología para disminuir ese costo y, eventualmente, generar una asignación óptima.

Como resulta evidente, el análisis de la elección del mejor instrumento de asignación se complicaría si el costo de control del daño y el costo marginal del daño resultan desconocidos, asimismo, si éstos se encuentran correlacionados positiva o negativamente, cuestión que no se abordará en el presente análisis.

\section{Gráfica 4 \\ Caso 2: acuífero con sobreexplotación extrema en condiciones de incertidumbre}

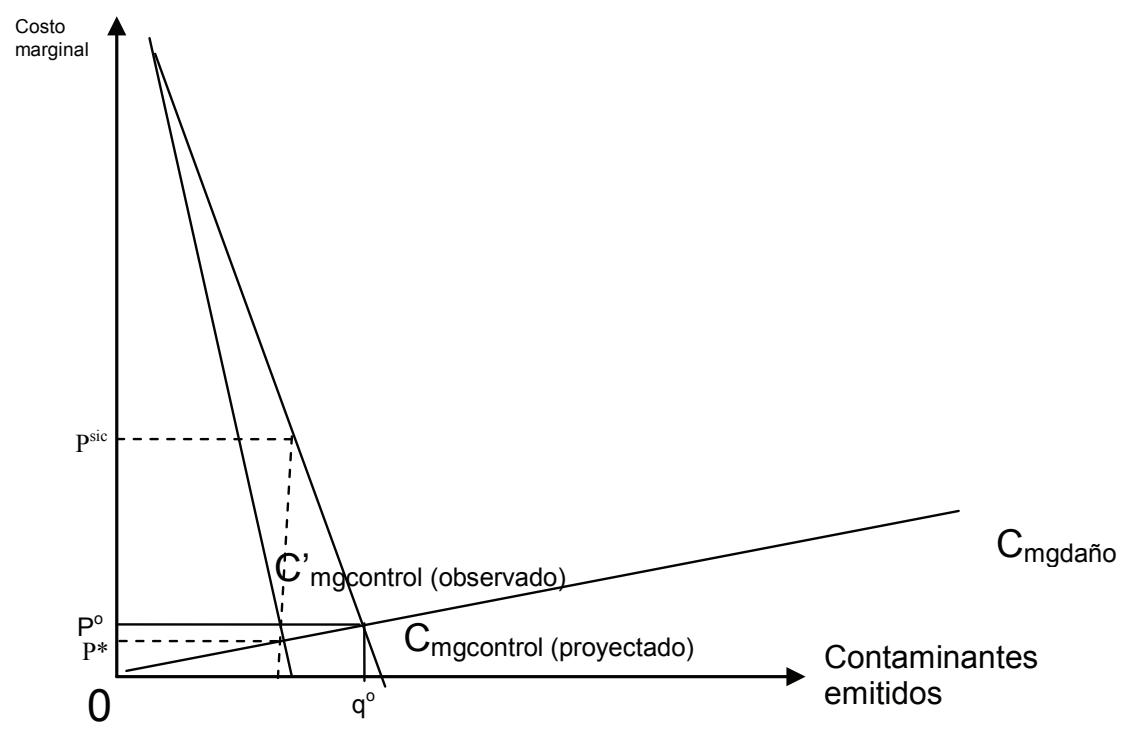

Fuente: Tietenberg (2006) 


\section{CONCLUSIONES}

En México existe una crisis por la sobreexplotación de acuíferos debida a dos fallas de políticas públicas: 1) la inexistencia de un mecanismo de asignación del agua subterránea vía precios que sea eficiente, sean directos con medición volumétrica o indirectos vía tarifas eléctricas para bombeo agrícola y 2) la ineficiencia en hacer cumplir el régimen de concesiones, asignaciones y permisos establecido en la Ley de Aguas Nacionales.

La crisis podría controlarse si se administra la demanda de agua, sin embargo, la autoridad del agua ha administrado históricamente la oferta a través de mayor construcción de infraestructura y aumento en el gasto, sin resolver los problemas de derechos de propiedad y sin hacer uso total de las facultades necesarias para realizar un óptimo cumplimiento de la ley. Por ejemplo, se ha hecho un mayor uso del régimen de control de las concesiones y zonas de veda y reglamentadas sin obtener los resultados esperados, ya que, recientemente, se han detectado altos niveles de abatimiento y deterioro de la calidad del agua en las zonas con mayor antigüedad de prohibición de extracción de aguas nacionales.

No se ha generado una transición hacia mecanismos de asignación vía precios, dado que los altos costos de instrumentación y transacción de los mecanismos de precios directos limitan su uso en México, aunado a que un gran porcentaje de agricultores no disponen de dispositivos de medición del agua que consumen y existe resistencia política a instalarlos.

Los incentivos de ahorro y uso eficiente del agua que pudiera tener el uso de precios indirectos, como la tarifa eléctrica para bombeo agrícola, son limitados debido a que las tarifas se encuentran fuertemente subsidiadas. Por su parte, los mercados de derechos de agua aún no se desarrollan plenamente pues requieren el establecimiento adecuado de los derechos de propiedad, garantía de cumplimiento y sanción de los contratos y bajos costos de transacción, para realizar los intercambios. 
Por otra parte, los agricultores mexicanos ejercen enormes presiones por mantener el status quo de un sistema mixto ineficiente, que combina un mecanismo de precios y tarifas, con subsidios altamente distorsionantes para el consumo de agua, con un mecanismo de asignación vía cantidades, debido a que esto les trae grandes beneficios, y cualquier reforma les representaría fuertes pérdidas, ya que su excedente del consumidor disminuiría con cualquier mecanismo de precios que se establezca. Además, tales beneficios se encuentran concentrados en un grupo limitado, mientras que los costos se encuentran dispersos en la sociedad y a través del tiempo.

Finalmente, en un escenario de incertidumbre e información incompleta, la distribución de las ganancias y costos por usar un mecanismo de precios o cantidades dependerá de la sensibilidad de los costos marginales del daño y de los costos de control del agua contaminada, debido a la sobreexplotación extrema a los diferentes niveles de extracción y/o contaminación. Así, en un escenario de cambio climático con altos niveles de incertidumbre en los niveles y tendencias de la precipitación, sequía y temperatura, el costo de equivocarse en la elección del instrumento de política puede tener grandes repercusiones en bienestar. Este riesgo refuerza la tendencia a no reformar la situación actual.

No obstante, aun con incertidumbre y altos costos políticos la reducción o transformación en la forma de entregar el subsidio existente en las tarifas eléctricas puede traer grandes ganancias en eficiencia y en la sustentabilidad de los acuíferos del país, especialmente los más sobreexplotados. La recomendación de política de las agencias de desarrollo, en este sentido, es desacoplar o desvincular el consumo de energía del subsidio otorgado vía precios de los insumos, es decir, se recomiendan medidas que tengan un efecto neutral en la asignación del recurso, tales como transferencias lump-sum a los agricultores al inicio del año para cubrir sus facturas estimadas de energía, de tal forma que reciban las señales de escasez relativa y del mayor costo de extracción de bombear agua de mayores profundidades, a través de sus tarifas eléctricas; a la vez que reciben el monto del subsidio, pero 
desacoplado o desvinculado del consumo. Este tipo de medidas son Pareto-eficientes, ya que disminuyen la sobreextracción de agua en los acuíferos, mantienen el estado actual de subsidios recibidos por los agricultores, la producción agrícola se vuelve sustentable y se podría mantener el equilibrio político al maximizar su aceptación entre los diferentes actores involucrados. 


\section{REFERENCIAS}

Aguilar, A. y R. Canizales. 2008. "La demanda de transmisión de derechos de agua en México: un análisis descriptivo para el periodo 20012006", Gaceta de Administración del Agua, II(1) (faltan páginas).

Auditoría Superior de la Federación. 2009. Informe del resultado de la revisión y fiscalización superior de la cuenta pública 2007, VI (2), marzo.

Barde, J.P. y O. Honkatukia. 2003. Environmentally Harmful Subsidies, OCDE, París.

Brubaker, Earl. 1997. "The Tragedy of the Public Budgetary Commons", The Independent Review, 1(3): 353-370.

Canizales, Rogelio. 2009. "Transmisión de derechos inscritos en el Registro Público de Derechos del Agua, 2008”, Reporte económico de administración del agua, primer trimestre.

Conagua. 2003. Estadísticas del Agua en México, México.

2008. Programa Nacional Hídrico, 2007-2012, México.

2010. Censos de aprovechamientos de los acuíferos del programa piloto para apoyar la estabilización de acuíferos sobreexplotados en México, Subdirección General de Administración del Agua, México.

2011. Agenda del agua 203o, México.

Dinar, A. 2000. The political economy of water pricing reforms, World Bank.

DOF. 2002. Ley de Energía para el Campo, México. 
(varios años). Acuerdo que autoriza el ajuste y reestructuración a las tarifas para suministro y venta de energía eléctrica, México. 7 de enero y 8 de agosto de 2003, y sus modificaciones de fechas 19 de julio de 2005, 11 de noviembre de 2005 y 21 de diciembre de 2007.

(varios años). Ley Federal de Derechos, México.

Easter, W., M. Rosegrant y A. Dinar. 1998. Markets for water: potential and performance, Massachussetts: Kluwer Academic Publishers.

Economic Research Service of the US Department of Agriculture. 2004. Decoupled Payments in a Changing Policy Setting, Agricultural Economic Report, núm. 838, noviembre.

FAO. 2007. Aquastat Country fact Sheet Brazil, en http://www.fao.org/ nr/water/aquastat/data/factsheets/aquastat_fact_sheet_bra_ en.pdf

Galindo, L.M. 2008. La economía del cambio climático en México, Semarnat-SHCP.

Guevara , Alejandro et. al. 2007. Políticas y Gasto Público en Energía Eléctrica para el Bombeo de Agua en Actividades Agrícolas, Reporte final para el Banco Interamericano para el Desarrollo, agosto.

INE-UACH, Instituto Nacional de Ecología y Universidad Autónoma de Chapingo. 2010. Encuesta a usuarios agrícolas de tarifas eléctricas, 2010, Resultados preliminares.

INEGI. 2007. Censo agrícola y ganadero, 2007. Resultados por localidad.

Kemper, Karin, S. Foster, H. Garduno, M. Nanni y A. Tuinhof. 2003. Economic Instruments for Groundwater Management - Using Incentives to Improve Sustainability, World Bank Briefing Note 7 , Banco Mundial. 
Muñoz Piña, C. 2007. "Análisis del subsidio a la tarifa o9”, en J.L. López Calva (comp.), Sustentabilidad y desarrollo ambiental. Agenda para el desarrollo, núm. 14, UNAM/Porrúa, México.

OCDE, 2003, Environmental Harmfully Subsidies: Policy, Issues and Challenges. París. En http://www.ine.gob.mx/descargas/dgipea/harmful_subsidies.pdf

Paquin, et al. (s/f). La reingeniería de subsidios en la agricultura: hacia políticas de desarrollo sustentable en los países de la OCDE y recomendaciones para México, INE-Semarnat. México.

Presidencia de la República. 2007. Plan Nacional de Desarrollo, 20072012, México.

Rivero, Edgar. y S. Nieto. 2011. ¿Cómo y por qué influyen los grupos de interés en las políticas ambientales?: El caso del Programa del pago por servicios ambientales hidrológicos, Documento de trabajo, S/N, INE.

Roemer, A. 2007. Economía y derecho: políticas públicas del agua, Porrúa, México.

Saleth, M. y A. Dinar. 2004. The Institutional Economics of Water, World Bank y EE Publishing.

Scott, C., T. Shah y S. Buechler. 2003. Energy Pricing and Supply of Groundwater Demand management: Lessons from Mexican agriculture. Anand, India: IWMI-TATA Water Policy Research Highlight núm. 3.

Shah, T. 2008. Governing the Groundwater Economy: Comparative Analysis of National Institutions and Policies in South Asia, China and Mexico, en V. Ballabh (comp.), Governance of Water: Institutional Alternatives and Political Economy, Sage Publications, India. pp. 237-266. 
Shah, T., D. Molden, R. Sakthivadivel y D. Seckler. 200o. The Global Groundwater Situation: Overview of Opportunities and Challenges, International Water Management Institute, Colombo, Sri Lanka

Tietenberg, T. 2006. Environmental and Natural Resource Economics, 7a ed. Pearson, NY.

Tsur et al. 2004. Pricing Irrigation Water: Principles and Cases from Developing Countries, RFF Press, Washington, D.C.

Weitzman, M. 1974. Prices vs Quantities, The Review of Economic Studies, 41(4).

Zilberman, D. y K. Schoengold. 2007. The Economics of Water, Irrigation and Development, en R. Evenson y P. Pingali (comps.), Handbook of Agricultural Economics, vol. 3, Elsevier Science, Holanda. 


\section{ANEXO}

\section{Cuadro A1}

Instrumentos de la política hídrica nacional que puede utilizar la Conagua de acuerdo con la Ley de Aguas Nacionales

a) Instrumentos regulatorios: Los más importantes son los títulos de concesión, sólo pueden otorgarse en caso de que exista disponibilidad del recurso y el acuífero no se encuentra en zona de veda. También entran en esta categoría los permisos, asignaciones y las prórrogas.

b) Instrumentos económicos: Son aquellos que están contenidos en la Ley Federal de Derechos, la cual establece el pago por el uso de aguas nacionales, así como por el uso de cuerpos receptores de las descargas de aguas residuales.

c) Instrumentos de orden y control: Los instrumentos básicos son las inspecciones, mediciones y las sanciones. La Conagua realiza visitas de inspección para monitorear y sancionar a los usuarios, para verificar el cumplimiento de las obligaciones establecidas en la Ley de Aguas Nacionales.

d) Instrumentos participativos: Los más destacados son los consejos de cuenca y las asociaciones de usuarios (i.e. Cotas). Los primeros se establecen para conciliar los intereses de los gobiernos federal, estatal o municipal con los usuarios y otros grupos interesados, en tanto que los segundos, se dan principalmente en los distritos y las unidades de riego. 
Edgar Rivero Cob • Helena García Romero

\section{Gráfica A1}

Estructura de las tarifas eléctricas para bombeo agrícola, 2010

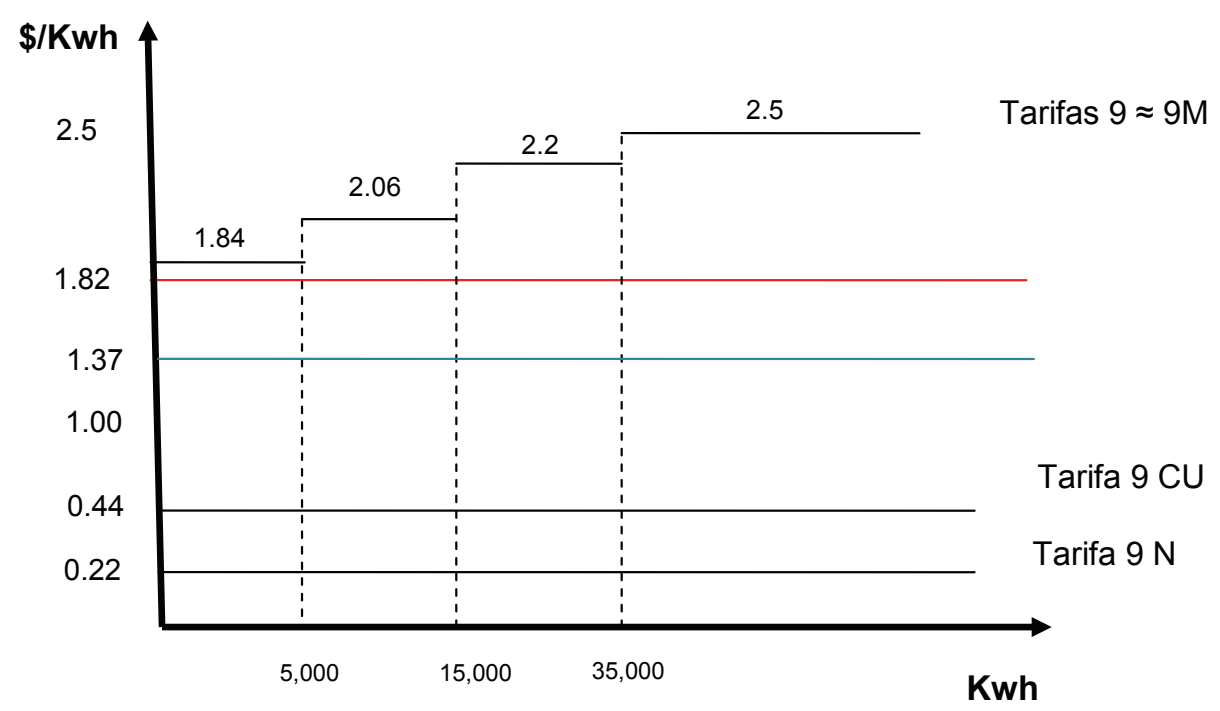

Fuente:1/ DOF, 7 de enero y 8 de agosto de 2003 y sus modificaciones, 2/DOF, 7-I-2003 y sus modificaciones, 3/ Energy Information Agency (EIA) 